Commun. Math. Phys. 145, 393-410 (1992)

Communications in

Mathematical

Physics

(C) Springer-Verlag 1992

\title{
Yang-Mills Fields which are not Self-Dual
}

\author{
Gil Bor \\ Department of Mathematics, University of Arizona, Tucson, AZ 85721, USA
}

Received July 19, 1991; in revised form November 18, 1991

\begin{abstract}
The purpose of this paper is to prove the existence of a new family of non-self-dual finite-energy solutions to the Yang-Mills equations on Euclidean four-space, with $S U(2)$ as a gauge group. The approach is that of "equivariant geometry:" attention is restricted to a special class of fields, those that satisfy a certain kind of rotational symmetry, for which it is proved that (1) a solution to the Yang-Mills equations exists among them; and (2) no solution to the self-duality equations exists among them. The first assertion is proved by an application of the direct method of the calculus of variations (existence and regularity of minimizers), and the second assertion by studying the symmetry properties of the linearized self-duality equations. The same technique yields a new family of nonself-dual solutions on the complex projective plane.
\end{abstract}

\section{Introduction}

The Yang-Mills functional (or "energy") is defined on the space of connections on the principal $S U$ (2)-bundle $\mathbb{R}^{4} \times S U(2)$ on Euclidean four-space $\mathbb{R}^{4}$ by assigning to a connection $A$ the $L^{2}$-norm of its curvature $F_{A}$

$$
Y M(A):=-\int_{\mathbb{R}^{4}} \operatorname{tr}\left(F_{A} \wedge * F_{A}\right),
$$

where "*" is the Hodge duality operator on 2 -forms on Euclidean $\mathbb{R}^{4}$. The corresponding variational equations are the Yang-Mills equations

$$
d_{A} * F_{A}=0,
$$

where $d_{A}$ is the covariant exterior derivative associated with the connection $A$. From the Bianchi identity, $d_{A} F_{A}=0$, it follows that if $A$ is (anti-)self-dual,

$$
F_{A}= \pm * F_{A}
$$

("+" for self-dual, "-" for anti-self-dual), then it satisfies the Yang-Mills equations.

We will be concerned in this paper only with finite-energy solutions to Eq. (1) and (2). An example of a nontrivial (i.e. with nonvanishing curvature) finite- 
energy solution to the self-duality equations (2) (and hence to the Yang-Mills equations (1)) is given by the formula

$$
A(x)=\operatorname{Im}\left(\frac{\bar{x} d x}{1+|x|^{2}}\right),
$$

where $x$ is a quaternionic variable on $\mathbb{R}^{4} \cong \mathbb{H}$, and we identify the Lie algebra of $S U(2) \cong S p(1)$ with the set of imaginary quaternions. In 1979, similar formulae were discovered for all finite-energy self-dual and anti-self-dual connections on Euclidean $\mathbb{R}^{4}$ (see Atiyah [2] for details).

It will be useful for us to replace $\mathbb{R}^{4}$ with its conformal compactification, the standard four-sphere $S^{4}$. By the conformal invariance of the Yang-Mills equations and energy (this follows from the conformal invariance of the * operator on 2-forms in four dimensions) solutions on the four-sphere give rise, via stereographic projection, to finite-energy solutions on Euclidean space. The converse, although not used in this paper, is also true, by a theorem of $\mathrm{K}$. Uhlenbeck: a finite-energy Yang-Mills connection on $\mathbb{R}^{4}$ can be "extended at infinity," i.e., comes from a Yang-Mills connection on some principal bundle, non-trivial in general, on $S^{4}$. Finite-energy Yang-Mills theory on Euclidean $\mathbb{R}^{4}$ is thus equivalent to Yang-Mills theory on $S^{4}$. For example, Formula (3) corresponds to the homogeneous connection on the quaternionic Hopf bundle $S^{7} \rightarrow S^{4}$.

As is well known, the space of connections on $S^{4}$ breaks into countably many connected components labeled by the second Chern number

$$
c_{2}=\frac{1}{8 \pi^{2}} \int_{S^{4}} \operatorname{tr}\left(F_{A} \wedge F_{A}\right),
$$

and the self-dual and anti-self-dual connections in each component form the absolute minima of the Yang-Mills functional [2].

Until recently, the only known finite-energy solutions to the Yang-Mills equations were the self-dual and anti-self-dual solutions (hereafter, just "selfdual" for simplicity). C. Taubes showed in [14] that all finite-energy Yang-Mills solutions on Euclidean $\mathbb{R}^{4}=\mathbb{R}^{1} \times \mathbb{R}^{3}$ with the $O$ (3) "spatial" symmetry are necessarily self-dual. J. Bourguignon and B. Lawson have shown [7] that non-selfdual solutions must be unstable (in the sense of having energy decreasing perturbations) and C. Taubes gave also a lower bound on their Morse index [15]. T. Parker has shown [9] that $\mathbb{R}^{4}=\mathbb{C}^{2}$ with the $U(2)$-symmetry admits non-selfdual solutions provided the standard metric on $\mathbb{R}^{4}$ is appropriately perturbed.

In 1989, L. Sibner, R. Sibner, and K. Uhlenbeck published a proof for the existence of some non-self-dual solutions on Euclidean $\mathbb{R}^{4}$ [13]. In their proof, modeled on a previous proof of $\mathrm{C}$. Taubes for the existence of non-self-dual solutions to the Euclidean monopole equations, solutions arise as minmax elements associated with loops of connections with a certain circle symmetry. Selfdual solutions (called hyperbolic monopoles in this case) are excluded by appropriate energy estimates.

In this paper we establish the existence of a new family of finite-energy nonself-dual solutions (different from the ones in [13]) by restricting attention, as in [13], to a class of symmetric connections, but with a rotational ( $S O(3))$ symmetry of a certain kind. For this symmetry, $S^{4}$ is thought of as the unit sphere in the 5 dimensional space consisting of real symmetric traceless $3 \times 3$ matrices with $S O(3)$ 


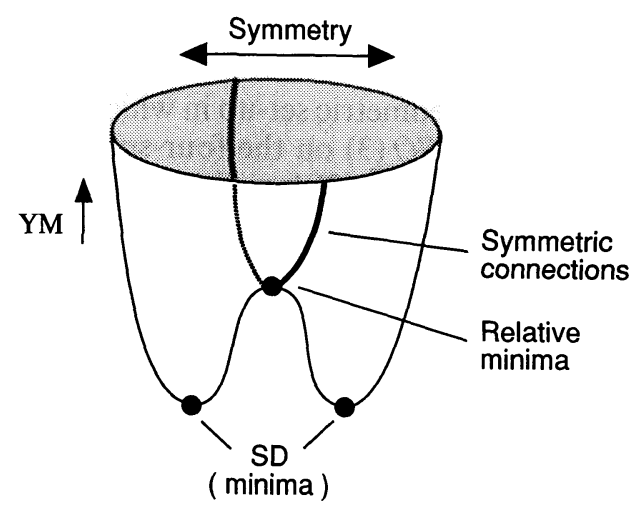

Fig. 1. Non-minimal symmetric critical points of the Yang-Mills functional

acting by matrix conjugation. We first identify the connected components of the space of symmetric connections, that is, connections on $S^{4}$ which are invariant under our $S O$ (3) symmetry (there are infinitely many such components). We are then able to exclude self-dual connections from most connected components by showing that the self-duality equations, linearized at a symmetric solution, do not possess the required symmetry; therefore, any symmetric solution to the YangMills equations is bound to be non-self-dual and so we look for a (symmetric) minimum of the variational problem. This, we prove, exists as a limit of a minimizing sequence of symmetric connections (see Fig. 1).

It is instructive to compare our method of proof with that of Sadun and Segert in [12]. Both begin the same way, by characterizing the equivariant bundles with the given $S O$ (3) action on $S^{4}$. Sadun and Segert then proceed by reducing the Yang-Mills equations by the full symmetry group. This leads to a system of ordinary differential equations on an interval with certain singularities at the endpoints. (This ODE approach was initiated by Urakawa in [16], advanced by Bor and Montgomery in [6] and completed by Sadun and Segert.) The main technical difficulty with this approach concerns the behavior of possible solutions near the singular endpoints and this involves a significant amount of work. In contrast, our use of normal slices near singular orbits essentially reduces the YangMills equations to an elliptic PDE in two variables (maintaining a "residual" symmetry coming from the isotropy group at a singular orbit), but now with no singularities. This simplifies much of the analysis. Two other new features of our work are (1) a simple method for excluding self-dual solutions out of our list of equivariant solutions. This method is based on the Atiyah-Bott fixed-point formula; and (2) our method easily generalizes to deal with a similar class of equivariant solutions over $\mathbb{C} P^{2}$ (the complex projective plane with its standard Fubini-Study metric. See Remark 4 at the end of Sect. 3). T. Parker has also obtained recently results overlapping with the ones given here [10].

Following this introduction, Sect. 1 describes the $S O(3)$-action with which we work and the lifts of the action to principal $S U(2)$-bundles. In Sect. 2 we prove that each lift (there are infinitely many) admits Yang-Mills solutions, and in Sect. 3 we prove that most lifts do not admit self-dual solutions. The appendix contains several simple facts of a general nature concerning the local structure of equivariant bundles. 


\section{A Family of Equivariant Bundles}

In this section we introduce the geometric set-up in which we work. We first define an action of the rotation group $S O(3)$ on the four-sphere $S^{4}$. We then look for "lifts" of this action to principal $S U(2)$-bundles over $S^{4}$. The result (Proposition 1.4) is a family of bundles with an action of $S \widetilde{O(3)}$ (the simply-connected double-cover of $S O(3))$ covering the $S O(3)$-action on $S^{4}$.

\subsection{The SO(3)-Action on $S^{4}$}

The space of all $3 \times 3$ real symmetric traceless matrices is a 5-dimensional real vector space $\cong \mathbb{R}^{5}$. The rotation group $S O(3)$ acts on this space by matrix conjugation

$$
x \mapsto s x s^{-1}, \quad x \in \mathbb{R}^{5}, s \in S O(3),
$$

preserving the norm $\|x\|^{2}=\operatorname{tr}\left(x^{2}\right)$, so that the $S O$ (3)-action on $\mathbb{R}^{5}$ restricts to an action by isometries on the unit sphere $S^{4}=\{x \mid\|x\|=1\} \subset \mathbb{R}^{5}$. We now list some of the properties of this action.

Proposition 1.1. 1. The set of diagonal matrices in $S^{4}$ of the form

$$
\Sigma:=\left\{\left(\begin{array}{ccc}
\lambda_{1} & 0 & 0 \\
0 & \lambda_{2} & 0 \\
0 & 0 & \lambda_{3}
\end{array}\right) \mid \lambda_{1} \geqq \lambda_{2} \geqq \lambda_{3}, \lambda_{1}+\lambda_{2}+\lambda_{3}=0, \lambda_{1}^{2}+\lambda_{2}^{2}+\lambda_{3}^{2}=1\right\} \subset S^{4}
$$

is a global section: every orbit of the $S O(3)$-action on $S^{4}$ intersects $\Sigma$ at precisely one point.

2. $\Sigma$ forms a $\pi / 3$-arc of a great circle on $S^{4}$, whose endpoints, denoted by $\sigma_{+}$and $\sigma_{-}$, are given by

(see Fig. 2).

$$
\sigma_{+}:=\frac{1}{\sqrt{6}}\left(\begin{array}{ccc}
1 & 0 & 0 \\
0 & 1 & 0 \\
0 & 0 & -2
\end{array}\right), \quad \sigma_{-}:=\frac{1}{\sqrt{6}}\left(\begin{array}{ccc}
2 & 0 & 0 \\
0 & -1 & 0 \\
0 & 0 & -1
\end{array}\right)
$$

Proof. 1. By a well known fact of linear algebra, every symmetric matrix is conjugate, by an orthogonal matrix, to a diagonal matrix, unique up to the order of its diagonal elements.

2. The set of diagonal matrices in $S^{4}$ is the intersection of a 2-dimensional subspace in $\mathbb{R}^{5}$ with $S^{4}$, and thus a great circle. The condition $\lambda_{1} \geqq \lambda_{2} \geqq \lambda_{3}$ defines a $60^{\circ}$-arc on this circle.

Fig. 2. The global section $\Sigma$ on $S^{4}$

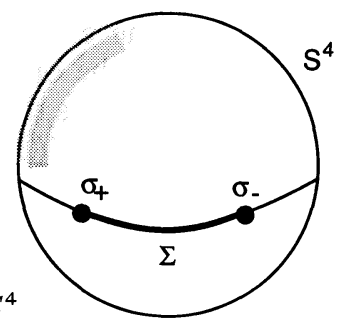


We recall that the stability group at a point $x$ in $S^{4}$ is, by definition, the set of elements in $S O$ (3) which fix $x$. The next proposition is obtained by a straightforward calculation.

Proposition 1.2. The stability groups along $\Sigma$ are (see Fig. 3)

- at the endpoint $\sigma_{+}$

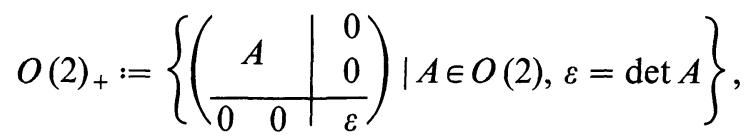

- at the endpoint $\sigma_{-}$

$$
O(2)_{-}:=\left\{\left(\begin{array}{c|cc}
\varepsilon & 0 & 0 \\
\hline 0 & A
\end{array}\right) \mid A \in O(2), \varepsilon=\operatorname{det} A\right\},
$$

- at each interior point of the arc $\Sigma$

$$
\begin{gathered}
\Gamma:=\left\{\left(\begin{array}{ccc}
\varepsilon_{1} & 0 & 0 \\
0 & \varepsilon_{2} & 0 \\
0 & 0 & \varepsilon_{3}
\end{array}\right) \mid \varepsilon_{i}= \pm 1, \varepsilon_{1} \varepsilon_{2} \varepsilon_{3}=1\right\} \\
O(2)_{+} \quad \Gamma \quad O_{-}^{(2)_{-}}
\end{gathered}
$$

Fig. 3. The stability groups along $\Sigma$

In the next proposition we introduce a cover of $S^{4}$ by two open sets.

Proposition 1.3. Let $\mathbb{R} P_{+}^{2}$ (respectively $\mathbb{R} P_{-}^{2}$ ) be the singular orbit through $\sigma_{+}$ (respectively $\sigma_{-}$), and let $N_{+}:=S^{4} \backslash \mathbb{R} P_{-}^{2}$ (respectively $N_{-}:=S^{4} \backslash \mathbb{R} P_{+}^{2}$ ). Then $N_{+}$ is a tubular neighborhood of $\mathbb{R} P_{+}^{2}$, i.e., it is $S O$ (3)-diffeomorphic to an $S O$ (3)homogeneous disk bundle over $\mathbb{R} P_{+}^{2}$. The analogous statement holds for $N_{-}$.

Proof. The required diffeomorphism, say for $N_{+}$, is given by the restriction of the exponential map $T S^{4} \rightarrow S^{4}$ to the $\pi / 3$-disk bundle in the normal bundle of $\mathbb{R}_{+}^{2}$.

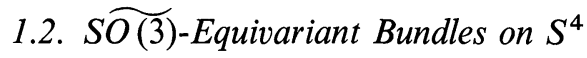

Here we seek to extend, or "lift," the $S O(3)$-action on $S^{4}$ to principal $S U(2)$ bundles over $S^{4}$. That is, we are looking for a principal $S U(2)$-bundle $P \rightarrow S^{4}$ together with a subgroup of bundle automorphisms of $P$ covering the given $S O(3)$-action on $S^{4}$.

\section{Examples.}

1. $P=S^{4} \times S U(2)$ with trivial action on the $S U$ (2)-factor (the "trivial lift").

2. $P=S^{4} \times S U(2)$ with $S \widetilde{O(3)}$ (the simply connected double-cover of $S O(3)$ ) acting on $S U(2)$ by left translations via the standard identification $S \widetilde{O(3)} \cong S U(2)$. 
3. $P=S^{7} \rightarrow \mathbb{H} P^{1} \cong S^{4}$ the quaternionic Hopf bundle, with $S \widetilde{O(3)}$ acting on $S^{7} \subset \mathbb{C}^{4}$ via the 4-dimensional irreducible complex representation. It is easily checked that the induced action on $S^{4}$ is isomorphic to our $S O$ (3)-action. There are actually two isomorphisms; they differ by the antipodal map $x \mapsto-x$ on $S^{4}$. A choice of one of them can be used to fix an orientation on our $S^{4}$. We come to this later in the section when we calculate Chern numbers.

We now explain how to obtain all possible lifts of the $S O$ (3)-action on $S^{4}$ to action of the double-cover $S \overparen{O(3)}$ on a principal $S U(2)$-bundle on $S^{4}$.

Associated with a given lift $P \rightarrow S^{4}$ are two integer invariants $n_{+}$and $n_{-}$ defined as follows. For each of the singular orbits $\mathbb{R} P_{+}^{2}$ and $\mathbb{R} P_{-}^{2}$ in $S^{4}$, the stability group $\cong \widetilde{O(2)} \subset S \widetilde{O(3)}$ of a point on the orbit acts on the fiber above it. Fixing a point on the fiber, the right action of the structure group $S U$ (2) identifies the fiber with $S U(2)$ itself, so that $\widetilde{O(2)}$ acts by left translations through some homomorphism $\lambda: \overparen{O(2)} \rightarrow S U(2)$. Restricting to the circle subgroup $S \widetilde{O(2)} \subset \overparen{O(2)}$, we have (after possibly conjugating by some element in SU (2))

$$
\lambda: e^{i \theta} \mapsto\left(\begin{array}{cc}
e^{n i \theta} & 0 \\
0 & e^{-n i \theta}
\end{array}\right)
$$

for some unique non-negative integer $n$. Furthermore, it is readily shown that $n$ must be either odd or 0 for it to come from a homomorphism of $\widetilde{O(2)}$ (see the proof of Proposition 1.4 below). In this way, with each lift of the $S O$ (3)-action on $S^{4}$ to a principal $S U$ (2)-bundle, we associate a pair of integers $n_{+}$and $n_{-}$, positive odd or 0 , corresponding to the singular orbits $\mathbb{R} P_{+}^{2}$ and $\mathbb{R} P_{-}^{2}$ respectively.

Examples. In the above three examples, $\left(n_{+}, n_{-}\right)$is $(0,0)$ for the first example (the trivial lift), $(1,1)$ for the second example, and $(3,1)$ or $(1,3)$ in the third example, depending on the choice of identification with our $S^{4}$.

In the next proposition we show that the pair of integers $\left(n_{+}, n_{-}\right)$uniquely determines the associated lift.

Proposition 1.4. The above definition of the pair $\left(n_{+}, n_{-}\right)$defines a one-to-one correspondence between

(1) $S \widetilde{O(3)}$-isomorphism classes of principal $S U(2)-b u n d l e s ~ P \rightarrow S^{4}$ with a nontrivial action of $S O(3)$ by bundle automorphisms covering the given $S O(3)$-action on $S^{4}$, and

(2) pairs $\left(n_{+}, n_{-}\right)$of positive odd integers.

Proof. We use an equivariant version of the usual "clutching construction" for principal bundles over a sphere. We cover $S^{4}$ with the two "hemispheres" $N_{+}$and $N_{\text {- }}$ (the tubular neighborhoods of the singular orbits $\mathbb{R} P_{+}^{2}$ and $\mathbb{R} P_{-}^{2}$, see Proposition 1.3), classify lifts $P_{ \pm} \rightarrow N_{ \pm}$over each of the tubular nighborhoods $N_{ \pm}$, and "glue" equivariantly over the intersection $N_{+} \cap N_{-}$via an equivariant isomorphism $\left.\left.P_{+}\right|_{N_{+} \cap N_{-}} \rightarrow P_{-}\right|_{N_{+} \cap N_{-}}$(over the identity on $N_{+} \cap N_{-}$). The lifts over each of the tubular neighborhoods $N_{+}$and $N_{-}$are classified, up to an $S \widetilde{O(3)}$ isomorphism, by the conjugacy class of the holonomy representation $\lambda$ : $\widetilde{O(2)} \rightarrow S U(2)$ at a point on the corresponding singular orbit, (see Appendix A.2). A short calculation shows that these conjugacy classes are given by the following list of representatives: 
1. The unique extension (up to conjugacy) of the homomorphism (5), where $n$ is an odd positive integer.

2. The trivial homomorphism $\widetilde{O(2)} \rightarrow\{1\} \subset S U(2)$.

3. The determinant homomorphism $\widetilde{O(2)} \rightarrow O(2) \stackrel{\text { det }}{\longrightarrow}\{ \pm 1\} \subset S U(2)$.

(The last two are the extensions of the $n=0$ case in (5)).

An $S O(3)$-equivariant "glueing" of $P_{+}$and $P_{-}$over $N_{+} \cap N_{-}$is given by a $\Gamma$ equivariant isomorphism between $P_{+}$and $P_{-}$over the normal slice $\Sigma_{0}:=\Sigma \backslash\left\{\sigma_{+}, \sigma_{-}\right\}=\Sigma \cap N_{+} \cap N_{-}$(see Proposition 1.1 and 1.2 for the definitions of $\Sigma$ and $\Gamma$ ), i.e., a map $\Sigma_{0} \rightarrow S U(2)$ which conjugates $\left.\lambda_{+}\right|_{\Gamma}$ and $\left.\lambda_{-}\right|_{\Gamma}$. This restricts $\lambda_{+}$and $\lambda_{-}$to be both of either the first or second type in the list above. Furthermore, in the first case $\left(n_{+}\right.$and $n_{-}$are odd) the "glueing" isomorphism is unique up to a sign, which can be extended over say $N_{+}$, hence we get a unique lift (up to an $S \widetilde{O(3)}$-isomorphism); likewise in the second case $\left(\lambda_{+}\right.$and $\lambda_{-}$are trivial), we get a unique lift, the trivial lift.

We recall that principal $S U(2)$-bundles over $S^{4}$ (without any equivariance constraint) are classified topologically by an integer invariant [5]; this can be taken as the second Chern number: by definition, the integral over $S^{4}$ of the second Chern class of the rank 2 vector bundle $E=P \times_{S U(2)} \mathbb{C}^{2}$ associated with the basic representation of $S U(2)$ (see Formula (4) in the Introduction).

We now wish to calculate the second Chern number of each of the bundles constructed in the last proposition in terms of the integers $n_{+}$and $n_{-}$. To fix the sign, we need to choose an orientation on our $S^{4}$. One way to do it is by identifying the Hopf bundle $\left(c_{2}=-1\right)$ with the $\left(n_{+}=3, n_{-}=1\right)$ bundle (see the third example at the beginning of the section). With this convention adopted, we have:

Proposition 1.5. The second Chern number of an $S \widetilde{O(3)}$-equivariant bundle $P$ of type $\left(n_{+}, n_{-}\right)$in Proposition 1.4 , is given by

$$
c_{2}=\frac{n_{-}^{2}-n_{+}^{2}}{8} .
$$

Proof. We use an integration formula for equivariant Chern classes due to Atiyah and Bott [4]. Restrict the $S \widetilde{O(3)}$-action to a circle-subgroup in $S \widetilde{O(3)}$, say the circle subgroup (the identity component) of $\widetilde{O(2)_{+}}$, the stabilizer of $\sigma_{+}$(see Proposition 1.2). The fixed point set of this circle-action consists of two points, $\sigma_{+}$and its antipodal $-\sigma_{+}$. The integration formula in [4] gives

$$
c_{2}=\frac{1}{8 \pi^{2}}\left(\frac{\operatorname{tr}\left(X_{+}^{2}\right)}{e_{+}}+\frac{\operatorname{tr}\left(X_{-}^{2}\right)}{e_{-}}\right),
$$

where $X_{ \pm} \in$ End $\left(\mathbb{C}^{2}\right)$ is the generator of the circle-action in the fiber of $E$ over the fixed point $\pm \sigma_{+} \in S^{4}$, and $e_{ \pm}$is the (signed) product of the weights of the circleaction on $S^{4}$ linearized at $\pm \sigma_{+}$. The circle-action on $\mathbb{R}^{5}$ is given by the weights of the 5-dimensional representation; these are $0, \pm 2, \pm 4$. Restricted to $S^{4}$, the weights at each of the fixed points are $\pm 2, \pm 4$. This determines $e_{ \pm}$up to a sign. Now at $\sigma_{+}$the circle-action gives the standard orientation, at $-\sigma_{+}$the opposite one, so $e_{ \pm}= \pm 8$. Equation(5) implies

$$
X_{ \pm}=\left(\begin{array}{cc}
2 \pi i n_{ \pm} & 0 \\
0 & -2 \pi i n_{ \pm}
\end{array}\right) \text {, }
$$


so $\operatorname{tr}\left(X_{ \pm}^{2}\right)=-8 \pi^{2} n_{ \pm}^{2}$. The stated formula for the second Chern number now follows by substitution in Eq. (7).

We have thus constructed a family of non-trivial $S \widetilde{O(3)}$-equivariant $S U(2)$ bundles indexed by a pair of positive odd integers $\left(n_{+}, n_{-}\right)$, where the second Chern number is given by $c_{2}=\left(n_{-}^{2}-n_{+}^{2}\right) / 8$.

\section{Existence of Invariant Yang-Mills Solutions}

Here we prove that each of the $S \widetilde{O(3)}$-equivariant bundles constructed in the previous section admits a Yang-Mills connection: It is a connection which minimizes the Yang-Mills energy amongst all $S \widetilde{O(3)}$-invariant (henceforth, simply "invariant") connections on the bundle in question.

Theorem 2.1. For each of the $S \widetilde{O(3)-e q u i v a r i a n t ~ b u n d l e s ~} P \rightarrow S^{4}$ of Proposition 1.4, the Yang-Mills functional, restricted to the set of invariant connections on $P$, attains a minimum which is a smooth $\left(C^{\infty}\right)$ solution to the Yang-Mills equations.

Proof. Let $\left\{A_{i}\right\}$ be a minimizing sequence of invariant connections on $P$. That is,

$$
\lim _{i \rightarrow \infty} Y M\left(A_{i}\right)=\inf \{Y M(A) \mid A \text { is an invariant connection on } P\} .
$$

We will show that such a sequence must be bounded (in the $L_{1}^{2}$ Sobolev metric on the space of connections on $P$, see Appendix A.4 for the definition). The rest follows the standard argument of the "direct method of the calculus of variations:" boundedness implies compactness (in the weak $L_{1}^{2}$-topology), i.e. the sequence has a weakly convergent subsequence, and since the Yang-Mills functional is weakly lower-semicontinuous and the set of invariant connections is weakly closed (as a norm-closed affine subspace of the space of $L_{1}^{2}$-connections on $P$ ), the limit connection is a minimizer and therefore a critical point of the Yang-Mills functional restricted to the set of invariant connections (which is a Hilbert submanifold of the space of all $L_{1}^{2}$-connections so it makes sense to speak of critical points). But then, by the "principle of symmetric criticality" [11, Theorem 5.4], it is a critical point of the Yang-Mills functional on the space of all connections, i.e., a weak solution of the Yang-Mills equations, for which regularity is by now standard [17, Corollary 1.4]. Alternatively, the last step (regularity) can be easily proven here directly using the reduced Yang-Mills equations for invariant connections (they form an elliptic system in our case. See the remark after the proof of Lemma 2.3). The theorem thus follows from the following proposition.

Proposition 2.2. A set of invariant connections on $P$, on which the Yang-Mills functional is bounded, is bounded (in $L_{1}^{2}$ ).

Remark. For general connections (not necessarily invariant) the proposition holds (locally) provided the connections are allowed to be gauge transformed first (see Uhlenbeck [17]). The invariance assumption here eliminates the need to make a gauge transformation.

Proof of Proposition 2.2. Since the statement is local (see Appendix A.4), it is enough to prove it for each of the tubular neighborhoods $N_{+}$and $N_{-}$(defined in 
Proposition 1.3). In each such neighborhood ${ }^{1}$, all relevant data reduces to a slice, i.e. a normal disk $D$ at a point on the singular orbit $\cong S O(3) / O(2)$. Under this "reduction to the slice," all $S O$ (3)-equivariant data on a tubular neighborhood reduces to $O$ (2)-equivariant data on the slice $D$, where the relevant estimates become rather simple in our case. The proof proceeds in two steps:

1. Representation theory: Break the connection and curvature forms into their $O$ (2)-irreducible components. All but 3 of the 12 components of the connection form vanish.

2. Energy estimates: Show that the 3 remaining components of the connection form are bounded (in $L_{1}^{2}$ ) by the Yang-Mills energy (the $L^{2}$-norm of the curvature).

Step 1. Restricted to the slice $D, P$ has a standard $O(2)$-trivialization

$$
\left.P\right|_{D} \cong D \times S U(2),
$$

with $\widetilde{O(2)}$ acting diagonally, on $D$ by the slice representation $\widetilde{O(2)} \rightarrow O(2)$, and on $S U$ (2) by left translations via the holonomy representation $\lambda: \widetilde{O(2)} \rightarrow S U(2)$ (see Appendix A.2). The holonomy representation $\lambda$ is determined (up to conjugacy in $S U$ (2)) by its weight, $n$, an odd positive integer (see Proposition 1.4). Correspondingly, we have the $O$ (2)-trivialization ${ }^{2}$ ad $\left.P\right|_{D} \cong D \times s u$ (2) with $O$ (2) acting on $s u(2)$ by the composition of $\lambda$ with the adjoint representation. Under the $O(2)$ action

$$
T_{y} N=T_{y} D \oplus v_{y} D=\mathbb{R}_{(2)}^{2} \oplus \mathbb{R}_{(1)}^{2}, \quad y \in D,
$$

and

$$
s u(2)=\mathbb{R}^{1} \oplus \mathbb{R}_{(n)}^{2},
$$

where subscripts denote $O(2)$-weights, and $\mathbb{R}^{1}$ in (9) is the determinant representation $O(2) \rightarrow\{ \pm 1\} \subset \mathbb{R}$. The first summand in (8) is the tangent space to the slice, and the second summand is the normal tangent space. Corresponding to (8), the connection 1-form of an invariant connection on $N$ decomposes as (see Appendix A.3)

where

$$
A=\alpha+\phi,
$$

$$
\alpha \in \Gamma\left(\Lambda^{1}(D) \otimes s u(2)\right), \quad \phi \in \Gamma\left(v^{*}(D) \otimes s u(2)\right),
$$

and its curvature 2-form decomposes as

where

$$
F_{A}=F_{\alpha}+d_{\alpha} \phi+\psi
$$

$$
F_{\alpha}=d \alpha+\frac{1}{2}[\alpha, \alpha], \quad d_{\alpha} \phi=d \phi+[\alpha, \phi],
$$

and $\psi$ is given below.

Lemma 2.3. 1. The values of $\alpha$ lie in $\mathbb{R}^{1}$, and it has no radial part. That is,

in polar coordinates $(r, \theta)$ on $D$.

$$
\alpha=\alpha_{\theta}(r) d \theta
$$

\footnotetext{
1 Since the proof is essentially identical for $N_{+}$and $N_{-}$, we suppress throughout the subscripts + or - for simplicity

${ }^{2}$ Henceforth, we pass to $O(2)=\widetilde{O(2)} /\{ \pm 1\}$ instead of the double cover $\widetilde{O(2)}$, since we only deal with ad $P$ where $\{ \pm 1\}$ acts trivially
} 
2. The values of $\phi$ lie in $\mathbb{R}_{(1)}^{2} \otimes \mathbb{R}_{(n)}^{2}=\mathbb{R}_{(n+1)}^{2} \oplus \mathbb{R}_{(n-1)}^{2}$.

3. Let

$$
\phi=\left(\phi_{+}, \phi_{-}\right)
$$

be the corresponding decomposition of $\phi$. Then the term $\psi$ in (11) is given by

$$
\psi=\left|\phi_{-}\right|^{2}-\left|\phi_{+}\right|^{2}-4 \alpha_{\theta}-n \text {. }
$$

Proof. The first and second parts are a consequence of the invariance of the values of $\alpha$ and $\phi$ with respect to the stability group $\Gamma \subset O(2)$ at a generic point of $D$ (all points except the origin). The third part follows from the standard formula for the curvature of an invariant connection (formula (20) in Appendix A.3).

Remark. It follows immediately from the first part of the last lemma that $d^{*} \alpha \equiv 0$. Adding this identity to the Yang-Mills equations for $\alpha$ and $\phi$ makes them into an elliptic system; to see this it is enough to note that there are two equations, one with the leading term $d^{*} d \alpha$, the second with $d^{*} d \phi$. This gives us the regularity of invariant solutions refered to just before the statement of Proposition 2.2.

Step 2. Here we are to bound the $L_{1}^{2}$-norm of the components $\alpha$ and $\phi$ in Formula (10) in terms of the Yang-Mills action: The first term in Formula (11) bounds $\alpha$ (Lemma 2.4), the second term bounds $\phi$, except in the case $n=1$, where it only bounds the $\phi_{+}$component, and we use the third term $\psi$ and $\alpha$ to bound $\phi_{-}$ (Lemma 2.5).

Lemma 2.4. Any $O(2)$-invariant 1 -form $\alpha \in \Lambda^{1}(D)$ without a radial component satisfies the inequality (pointwise)

$$
|\alpha| \leqq \text { const }\|d \alpha\|_{L^{2}(D)}
$$

Proof. Let $C_{r}$ be the boundary circle of a disk $D_{r}$ of radius $r$ about the origin of $D$, then by Stokes' theorem and the Cauchy-Schwartz inequality

$$
2 \pi\left|\alpha_{\theta}(r)\right|=\left|\oint_{C_{r}} \alpha\right|=\left|\int_{D_{r}} d \alpha\right| \leqq\left(\int_{D_{r}}|d \alpha|^{2}\right)^{1 / 2}\left(\int_{D_{r}} 1\right)^{1 / 2} \leqq \text { const } r\|d \alpha\|_{L^{2}(D)},
$$

hence

$$
|\alpha|=\left|\frac{\alpha_{\theta}}{r}\right| \geqq \text { const }\|d \alpha\|_{L^{2}(D)} \text {. }
$$

Lemma 2.5. $\phi$ satisfies the inequality (pointwise)

$$
|\phi|^{2} \leqq \text { const }\left(|d \phi|^{2}+|\psi|^{2}+|\alpha|^{2}\right)+\text { const } .
$$

Proof. The $O(2)$-equivariance of

$$
\phi_{ \pm}: D_{(2)} \rightarrow \mathbb{R}_{(n \pm 1)}^{2}
$$

implies (using polar coordinates on $D$ )

$$
\partial_{\theta} \phi_{ \pm}=\frac{(n \pm 1)}{2} i \phi_{ \pm}
$$


where $i$ denotes $90^{\circ}$-rotation in $\mathbb{R}_{(n \pm 1)}^{2}$, hence

$$
\begin{aligned}
|d \phi|^{2} & =\left|\left(\partial_{r} \phi\right) d r+\left(\partial_{\theta} \phi\right) d \theta\right|^{2}=\left|\partial_{r} \phi\right|^{2}+\frac{(n+1)^{2}\left|\phi_{+}\right|^{2}+(n-1)^{2}\left|\phi_{-}\right|^{2}}{4 r^{2}} \\
& \geqq \text { const }\left((n+1)\left|\phi_{+}\right|^{2}+(n-1)^{2}\left|\phi_{-}\right|^{2}\right),
\end{aligned}
$$

so if $n \neq 1$ we are done, otherwise only the $\phi_{+}$component is bounded, and we use the explicit form of $\psi$ in Formula (12) to bound $\phi_{-}$:

$$
\left|\phi_{-}\right|^{2}=\psi+4 \alpha_{\theta}+\left|\phi_{+}\right|^{2}+1 \leqq \text { const }\left(|\psi|^{2}+|\alpha|^{2}+|d \phi|^{2}\right)+\text { const, }
$$

since $\phi_{+}$has already been bounded.

\section{Non-Existence of Invariant Self-Dual Solutions}

Our purpose here is to prove that the $S \widetilde{O(3)}$-equivariant bundles of Sect. 1, with $n_{+}$and $n_{-}$greater than 1 , do not admit self-dual or anti-self-dual connections. Together with the existence result of the previous section, this completes the proof of existence of Yang-Mills connections which are neither self-dual nor anti-selfdual on all such bundles. We note, by the formula $c_{2}=\left(n_{-}^{2}-n_{+}^{2}\right) / 8$ of Proposition 1.5, that this includes all bundles with second Chern number $c_{2} \neq \pm 1$

Theorem 3.1. Let $P$ be an $S \widetilde{O(3)}$-equivariant principal $S U(2)$-bundle over $S^{4}$ of type $\left(n_{+}, n_{-}\right)$, where $n_{+}=3,5,7, \ldots$ (i.e. we exclude those bundles where either $n_{+}$or $n_{-}$equals 1). Then there is no self-dual or anti-self-dual SO(3)-invariant connection on $P$.

Proof. Let $A$ be an $S \widetilde{O(3)}$-invariant connection on $P$. We will first show that if $n_{-}>1$, then $A$ cannot be self-dual. The approach is to study the $S O$ (3)-action on the space of solutions to the linearized self-duality equations at $A$.

We denote by $\Omega^{p}$ the space of ad $P$-valued $p$-forms on $S^{4}$ (i.e. sections of $\Lambda^{p} \otimes$ ad $P$ ), and by $\Omega_{-}^{2}$ the ad $P$-valued anti-self-dual 2-forms. Associated to the connection $A$ on $P$, we have the covariant exterior derivative $d_{A}: \Omega^{p} \rightarrow \Omega^{p+1}$, and $d_{A}^{-}: \Omega^{1} \rightarrow \Omega_{-}^{2}$ is $d_{A}$ followed by the projection $p_{-}=(1-*) / 2$ on the anti-self-dual part. If $A$ is self-dual, then $d_{A}^{-} d_{A}=p_{-} F_{A}=0$ and we get the so-called deformation complex for the self-dual connection $A$

$$
0 \rightarrow \Omega^{0} \stackrel{d_{A}}{\longrightarrow} \Omega^{1} \stackrel{d_{A}^{-}}{\longrightarrow} \Omega_{-}^{2} \rightarrow 0 .
$$

It is easy to verify that this complex is elliptic, hence its cohomology groups $H^{*}$ are finite-dimensional real vector spaces. Furthermore, the $S O(3)$-invariance of $A$ implies that the maps $d_{A}$ and $d_{A}^{-}$in the complex (13) commute with the $S O$ (3)action $^{3}$, so that the cohomology groups become $S O(3)$-representation spaces. The deformation complex was introduced in [5, Sect. 6], where it was also proved that for an irreducible connection $A$ on a positively curved self-dual manifold (such as $S^{4}$ ) $H^{2}=H^{0}=\{0\}$. In our case, $A$ cannot be reducible, as the following lemma shows.

${ }^{3}$ We can pass to $S O(3)=S \widetilde{O(3) /\{ \pm 1\}}$, since the center $\{ \pm 1\} \subset S \widetilde{O(3)}$ acts trivially on the bundles appearing in the deformation complex 
Lemma 3.2. The only $S \widetilde{(3)}$-equivariant bundles which admit a reducible self-dual connection are those with $n_{+}=n_{-}=1$ and $n_{+}=n_{-}=0$.

Proof. On $S^{4}$, a reducible self-dual $S U$ (2)-connection is flat (its curvature vanishes). Over each of the singular orbits $\mathbb{R} P_{+}^{2}$ and $\mathbb{R} P_{-}^{2}$, the bundle admits an invariant flat connection only if the derivative of the holonomy representation $\lambda_{*}: \overparen{o(2)} \rightarrow s u(2)$ extends to a homomorphism $\Phi=\lambda_{*} \oplus \phi: s \overparen{o(3)} \rightarrow s u(2)$ (see Formula (20) in Appendix A.3). This happens exactly when either $n_{ \pm}=0(\Phi \equiv 0)$, or $n_{ \pm}=1$ ( $\Phi$ is an isomorphism), corresponding to the obvious flat connections in Examples 1 and 2 respectively in Sect. 1.2.

We are now ready to calculate the character $\chi\left(H^{1}\right)$ of the $S O$ (3)-representation $H^{1}$, the remaining cohomology. For this, let us first fix a circle subgroup, say $S^{1} \subset O(2)_{+}$(see Proposition 1.2). Restricted to $S^{1}, \chi\left(H^{1}\right)$ is just a trigonometric polynomial, but not an arbitrary one: if $\chi\left(\mathbb{R}^{r}\right)$ is the character of the irreducible $r$-dimensional representation of $S O(3), r=1,3,5, \ldots$, then the coefficients $m_{r}$ in the decomposition

$$
\chi\left(H^{1}\right)=\sum_{r} m_{r} \chi\left(\mathbb{R}^{r}\right)
$$

must clearly be non-negative integers. We will now show that $m_{1}=-1$ when $n_{-}>1$, so that $A$ cannot be an $S O(3)$-invariant self-dual connection.

To calculate $\chi\left(H^{1}\right)$, we note that the fixed points of the $S^{1}$-action on $S^{4}, \sigma_{+}$and its antipodal $-\sigma_{+}$, are clearly nondegenerate, so we can apply the fixed point formula of Atiyah and Bott [3], which in our case reads (after taking into account the vanishing of $H^{2}$ and $\left.H^{0}\right)$,

$$
\chi\left(H^{1}\right)=-v_{-}-v_{+},
$$

where

$$
\begin{aligned}
& v_{ \pm}=\left(\chi_{ \pm}^{0}-\chi_{ \pm}^{1}+\chi_{ \pm}^{2}\right) / \delta_{ \pm}, \\
& \delta_{ \pm}=\operatorname{det}\left(I-L_{ \pm}\right) .
\end{aligned}
$$

Here, $\chi_{ \pm}^{i}, i=0,1,2$, denotes the character of the $S^{1}$-representation on the fiber over the fixed point $\pm \sigma_{+}$in the $i$-th bundle of the deformation complex (13), and $L_{ \pm}$is the linearized action at the fixed point $\pm \sigma_{+} \in S^{4}$. The weights of the $S^{1}-$ action at the fixed points for the various bundles involved are

\begin{tabular}{l|l|l|l} 
& ad $P$ & $\Lambda^{1}$ & $\Lambda_{-}^{2}$ \\
\hline \hline$\sigma_{+}$ & $0, \pm n_{+}$ & $\pm 1, \pm 2$ & $0, \pm 1$ \\
\hline$-\sigma_{+}$ & $0, \pm n_{-}$ & $\pm 1, \pm 2$ & $0, \pm 3$ \\
\hline
\end{tabular}

We denote $z=e^{i \theta}$ on $S^{1}$, and calculate from the weight table

$$
\begin{aligned}
\chi_{ \pm}^{0} & =1+z^{n_{ \pm}}+\bar{z}^{n_{ \pm}}, \\
\chi_{ \pm}^{1} & =\left(z+\bar{z}+z^{2}+\bar{z}^{2}\right) \chi_{ \pm}^{0}, \\
\chi_{+}^{2} & =(1+z+\bar{z}) \chi_{+}^{0}, \\
\chi_{-}^{2} & =\left(1+z^{3}+\bar{z}^{3}\right) \chi_{-}^{0}, \\
\delta & =\delta_{ \pm}=\left(z^{1 / 2}-\bar{z}^{1 / 2}\right)^{2}(z-\bar{z})^{2},
\end{aligned}
$$


so that substitution in Formula (15) yields

where

$$
\chi\left(H^{1}\right)=-Q / \delta,
$$

$$
Q=\left(2-z-\bar{z}-z^{2}-\bar{z}^{2}+z^{3}+\bar{z}^{3}\right)\left(1+z^{n_{-}}+\bar{z}^{n_{-}}\right)+\left(2-z^{2}-\bar{z}^{2}\right)\left(1+z^{n_{+}}+\bar{z}^{n_{+}}\right) \text {. }
$$

The first factor in the first summand is $-(z-\bar{z})^{2}(1-z-\bar{z})$, the first factor in the second summand is $-(z-\bar{z})^{2}$, hence

$$
\chi\left(H^{1}\right)=\left[(1-z-\bar{z})\left(1+z^{n-}+\bar{z}^{n_{-}}\right)+1+z^{n_{+}}+\bar{z}^{n_{+}}\right] /\left(z^{1 / 2}-\bar{z}^{-1 / 2}\right)^{2} .
$$

The coefficient $m_{1}$ in (14) can now be easily recovered by the Weyl integration formula [1, Theorem 6.1]

$$
\begin{aligned}
m_{1} & =-\frac{1}{2} \int_{S^{1}}\left(z^{1 / 2}-\bar{z}^{1 / 2}\right)^{2} \chi\left(H^{1}\right) \\
& =-\frac{1}{2} \int_{S^{1}}(1-z-\bar{z})\left(1+z^{n_{-}}+\bar{z}^{n_{-}}\right)+1+z^{n_{+}}+\bar{z}^{n_{+}},
\end{aligned}
$$

which picks the constant term in the last integrand, giving

$$
m_{1}=\left\{\begin{array}{ll}
0 & n_{-}=1 \\
-1 & \text { else }
\end{array} .\right.
$$

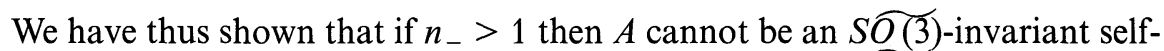

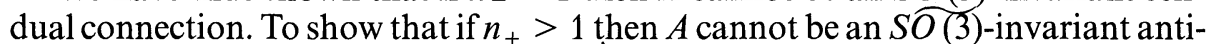
self-dual connection, we can either repeat the argument above with the obvious necessary modifications, or alternatively, use the fact that the antipodal map $x \mapsto-x$ on $S^{4}$ has an $S \widetilde{O(3)}$-equivariant lift which interchanges $n_{+}$and $n_{-}$. Since it is orientation reversing on $S^{4}$, it transforms an anti-self-dual connection with $n_{+}>1$ into a self-dual connection with $n_{-}>1$, so we are reduced to the case which has already been studied. This completes the proof.

Remarks. 1. With some more effort, one can compute in the case $n_{-}=1$ (for selfdual connections)

$\chi\left(H^{1}\right)=\chi\left(\mathbb{R}^{5}\right)+\chi\left(\mathbb{R}^{7}\right)+\chi\left(\mathbb{R}^{9}\right)+\ldots+\chi\left(\mathbb{R}^{\left(2 n_{+}-1\right)}\right), \quad n_{+}=3,5,7, \ldots$.

2. In the case $n_{-}=1$, the fact that $m_{1}=0$ means that self-dual $S \widetilde{O(3)}$-invariant connections, if they exist, are isolated. This is because the cohomology $H^{1}$ represents the tangent space to the moduli space of self-dual connections, and $m_{1}$ is the dimension of tangent space within $H^{1}$ to the space of invariant connections. In the case $\left(n_{+}, n_{-}\right)=(3,1)$ (the Hopf bundle), the (unique) $S \widetilde{O(5)}$-invariant self-

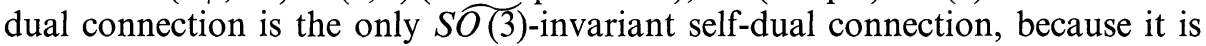
known that any other self-dual connection has a well-defined center (where the

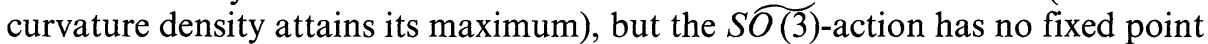
on $S^{4}$. Using the so-called ADHM construction, it is possible to show the existence of a unique self-dual $S \widetilde{O(3)}$-invariant connection for all the $\left(n_{+}, 1\right)$ bundles.

3. We do not know yet if there exist non-self-dual solutions with $c_{2}= \pm 1$. If not, one may ask if there is a sense in which any non-self-dual solution can be decomposed into self-dual and anti-self-dual parts. 
4. Taking the standard action of $S O(3)$ on $\mathbb{C}^{3}=\mathbb{R}^{3} \otimes \mathbb{C}$, we obtain an $S O$ (3) action on the complex projective plane $\mathbb{C} P^{2}$, for which we can look for non-selfdual connections by the same method we used for $S^{4}$. The details are very similar to the $S^{4}$ case so we give here only the conclusions. The lifts to principal $S U(2)$ bundles are classified by two non-negative integers $\left(n_{-}, n_{+}\right)$, where either both are odd, or $n_{-}=0$ and $n_{+}$is even. The second Chern number is given by $\left(n_{-}^{2}-n_{+}^{2}\right) / 4$, and non-self-dual connections occur for those lifts for which $n_{ \pm} \geqq 3$.

\section{Appendix}

Here we collect some simple useful facts of a general nature concerning the local structure of a principal bundle with a compact group action ${ }^{4}$.

\section{A.1. Tubular Neighborhoods and the Slice Representation}

Let $M$ be a Riemannian manifold and $S$ a compact Lie group acting on $M$ by isometries. Let $x$ be a point in $M$, and $S \cdot x$ the $S$-orbit through $x$. Then a small enough invariant neighborhood $N$ of the orbit $S \cdot x$ is a tubular neighborhood, i.e., a neighborhood of $S \cdot x$ which is $S$-isomorphic to a homogeneous vector bundle over $S \cdot x$. In fact, it is easy to see that for a sufficiently small $\varepsilon>0$, the exponential map restricted to the $\varepsilon$-disk bundle in the normal bundle $v(S \cdot x)$ defines such an isomorphism. The normal disk at $x, i: D_{x} \hookrightarrow N$ (i.e., the exponential image of the $\varepsilon$-disk in the space $v_{x}(S \cdot x)$ of normal vectors to $S \cdot x$ at $x$ ) is called a (normal) slice at $x$, and the action of the stability group $S_{x}:=\{s \in S \mid s \cdot x=x\}$ on $D_{x}$ is called the slice representation. As a homogeneous bundle, $N$ is $S$-isomorphic to the associated bundle $D_{x} \rightarrow S \times_{S_{x}} D_{x} \rightarrow S / S_{x} \cong S \cdot x$. In other words, the slice representation $\left(D_{x}, S_{x}\right)$ is a complete invariant for the extension of the $S$-action from $S \cdot x$ to $N$. In what follows, we shall see other instances of "reduction" of $S$ equivariant local data in a neighborhood of an orbit $S \cdot x$ to $S_{x}$-equivariant data on the slice $D_{x}$.

\section{A.2. Equivariant Principal Bundles}

Let $P \rightarrow N$ be an $S$-equivariant principal $G$-bundle (i.e. the $S$-action on $P$ commutes with the $G$-action, and the projection $P \rightarrow N$ is $S$-equivariant), where $N$ is a tubular neighborhood of an orbit $S \cdot x$. The action of the stability group $S_{x}$ on the fiber $P_{x}$ over $x$ is called the holonomy representation (at $x$ ). Fixing a point $p$ on the fiber $P_{x}$, the $G$-action identifies $P_{x}$ with $G$ itself, so that $S_{x}$ acts by left translations via a homomorphism $\lambda: S_{x} \rightarrow G$, defined by $s \cdot(p g)=p(\lambda(s) g)$. The holonomy representation is a complete invariant for extending (or "lifting") the $S$ action from $N$ to $P$. To see this, we proceed in two steps: (1) lift over $S \cdot x$; and (2) extend over $N$. Over $S \cdot x, P$ is a homogeneous bundle and so is canonically $S$ isomorphic to the associated bundle $P_{x} \rightarrow S \times_{S_{x}} P_{x} \rightarrow S / S_{x}=S \cdot x$. Over $N, P$ is $S$-isomorphic to the pull-back of the restriction $\left.P\right|_{S \cdot x}$ by the $S$-equivariant

4 Throughout this appendix, all group actions, functions, bundles etc., are assumed to be $C^{\infty}$ (infinitely differentiable) 
projection $N \rightarrow S \cdot x$. This follows from the more general standard fact that $S$ homotopic maps define $S$-isomorphic bundles; we apply this to the projection $N \rightarrow S \cdot x \subset N$ and the identity map $N \rightarrow N$, with the obvious homotopy along geodesics normal to $S \cdot x$. In particular, over the normal slice $D_{x}, P$ has the canonical $S_{x}$-trivialization

$$
\left.P\right|_{D_{x}} \cong D_{x} \times P_{x},
$$

with $S_{x}$ acting diagonally on $D_{x}$ by the slice representation, and on $P_{x} \cong G$ by left translations via the holonomy representation $\lambda: S_{x} \rightarrow G$.

The group of $S$-equivariant automorphisms of $P \rightarrow N$ (diffeomorphisms of $P$, over the identity on $N$, which commute with the actions of both $G$ and $S$ ) is represented on the slice $D_{x}$, using (16), by $S_{x}$-equivariant maps $D_{x} \rightarrow G$; that is, maps $D_{x} \rightarrow G_{\lambda}$, where $G_{\lambda}:=\left\{g \in G \mid g \lambda(s)=\lambda(s) g\right.$, for all $\left.s \in S_{x}\right\}$ is the centralizer of $\lambda$ in $G$. This is useful for $S$-equivariant "glueing" of bundles over different tubular neighborhoods.

\section{A.3. Invariant Connections}

Let $A$ be an $S$-invariant connection on an $S$-equivariant principal $G$-bundle $P \rightarrow N$, where $N$ is a tubular neighborhood of an orbit $S \cdot x$ with a normal slice $i$ : $D_{x} \hookrightarrow N$. The reduction of the connection $A$ to the slice $D_{x}$ consists of two pieces of data. The first piece is simply the restriction $\alpha=i^{*} A$ of $A$ to $i^{*} P=\left.P\right|_{D_{x}}$. The second piece, representing $A$ in the normal directions to $D_{x}$, is somewhat lengthier to describe. We associate with $A$ an $S$-equivariant map $\Phi$ of the Lie algebra of $S$ into the space of sections of the adjoint bundle ad $P=P \times{ }_{G} \mathfrak{g}$,

$$
\Phi: \mathfrak{s} \rightarrow \Gamma(\operatorname{ad} P),
$$

defined as follows. If $X \in \mathfrak{s}$, and $\tilde{X}$ is the induces vector field on $P$, then we set $\Phi(X):=A(\tilde{X})$. In other words, $\Phi(X)$ is the projection of $\tilde{X}$ onto the vertical bundle along the horizontal distribution defined by $A$. The $G$-invariance of $\tilde{X}$ together with the $G$-equivariance of $A$ ensure that the vertical vector field $A(\tilde{X})$ is $G$-equivariant, i.e. a section of ad $P$. The $S$-invariance of $A$ implies the $S$ equivariance of $\Phi$. Here, $S$ acts on $\mathfrak{s}$ by the adjoint representation, and on ad $P$ by the action induced from $P$. Next, we fix a complement $m$ to $\mathfrak{s}_{x}$, the Lie algebra of $S_{x}$; that is,

$$
\mathfrak{s}=\mathfrak{s}_{x} \oplus \mathfrak{m}
$$

is an $\mathrm{Ad}_{S_{x}}$-invariant decomposition of $\mathfrak{s}$. For example, we can take the orthogonal complement of $\mathfrak{s}_{x}$ with respect to an Ad-invariant inner product on $\mathfrak{s}$. The restriction of $\Phi$ to $m$ and $D_{x}$, denoted by $\phi$, forms the second piece of data in the reduction of the connection $A$ to the slice $D_{x}$. Thus $\phi$ is an $S_{x}$-invariant section of $\operatorname{ad}\left(\left.P\right|_{D_{x}}\right) \otimes \mathrm{m}^{*}$.

The decomposition (17) induces a natural trivialization of the normal bundle,

$$
v\left(D_{x}\right) \cong D_{x} \times m,
$$

as follows: we assign to a point $y \in D_{x}$ and a vector $X \in \mathrm{m}$ the normal component of the vector field $\tilde{X}$ generated by $X$ at $y$. This is indeed an isomorphism: it is enough to check that if the normal component of $\tilde{X}$ vanishes at $y \in D_{x}$ then $X=0$; indeed, if $\tilde{X}$ is tangent to $D_{x}$ at $y$, then the equivariance of the projection $N \rightarrow S \cdot x$ implies that $\tilde{X}$ vanishes at $x$, hence $X \in \mathfrak{s}_{x} \cap \mathfrak{m}=\{0\}$. 
The curvature $F_{A}$ is an $S$-invariant section of $\Lambda^{2}(N) \otimes$ ad $P$. In terms of the $S_{x}$ decomposition $\left.T N\right|_{D_{x}}=T D_{x} \oplus v\left(D_{x}\right)$, and the trivialization (18) of $v\left(D_{x}\right)$,

$$
\left.\Lambda^{2}(N)\right|_{D_{x}}=\Lambda^{2}\left(D_{x}\right) \oplus \Lambda^{1}\left(D_{x}\right) \otimes \mathrm{m}^{*} \oplus \Lambda^{2} \mathrm{~m}^{*}
$$

and the corresponding decomposition of $F_{A}$ is

$$
F_{\alpha}+d_{\alpha} \phi+\psi
$$

where $F_{\alpha}$ is the curvature of the connection $\alpha, d_{\alpha}$ is the associated covariant derivative, and $\psi$ is given by the standard formula (see, for example, Proposition 11.4 in Chapter II of [8])

$$
2 \psi(X, Y)=[\Phi X, \Phi Y]-\Phi[X, Y] \quad X, Y \in \mathrm{m} .
$$

We summarize the "reduction to the slice" in terms of the canonical trivialization (16): A lift of the $S$-action is given by the holonomy representation $\lambda$ : $S_{x} \rightarrow G$. An $S$-invariant connection $A$ is given by a pair $(\alpha, \phi)$, where $\alpha$ is an $S_{x^{-}}$ invariant $\mathrm{g}$-valued 1-form on $D_{x}$ and $\phi$ is an $S_{x}$-invariant $\mathrm{g} \otimes \mathrm{m}^{*}$ valued function on $D_{x}$. The curvature $F_{A}$ is given by the triple $\left(F_{\alpha}, d_{\alpha} \phi, \psi\right)$, where $F_{\alpha}=d \alpha+\frac{1}{2}[\alpha, \alpha]$, $d_{\alpha} \phi=d \phi+[\alpha, \phi]$, and $\psi$ is given by Formula (20), where $\Phi=\Phi_{0} \oplus \phi$, and $\Phi_{0}$ : $D_{x} \rightarrow \mathfrak{g} \otimes \mathfrak{s}_{x}^{*}$ is given by $\Phi_{0}(X)=\alpha(\tilde{X})+\lambda_{*}(X), X \in \mathfrak{s}_{x}$.

\section{A.4. Sobolev Spaces of Invariant Sections}

Let $U$ be an open set in Euclidean space. The Sobolev $L_{1}^{2}$-norm of a smooth function $f$ on $U$ is defined by

$$
\|f\|_{L_{1}^{2}(U)}:=\left(\int_{U}|f|^{2}+\sum_{i}\left|\frac{\partial f}{\partial x_{i}}\right|^{2}\right)^{1 / 2}=\left(\|f\|^{2}+\|d f\|^{2}\right)^{1 / 2}
$$

$\left(\|\cdot\|\right.$ stands for the ordinary $L^{2}$-norm on $\left.U\right)$. The set of functions in $C^{\infty}(U)$ with a finite $L_{1}^{2}$-norm forms a linear subspace, whose completion with respect to this norm is a Hilbert space denoted by $L_{1}^{2}(U)$.

Similarly, if $E$ is a metric vector bundle with a connection $A$ over a Riemannian manifold $M$, the $L_{1}^{2}$-norm is defined on sections of $E$ by replacing the derivative $d$ in (21) with the covariant derivative $d_{A}$. If another choice of connection $A^{\prime}$ is made, such that the difference $\alpha=A-A^{\prime} \in \Lambda^{1}(U) \otimes$ End $(E)$ is bounded (for example, if $M$ is compact), then $A^{\prime}$ gives an equivalent norm:

$$
\begin{aligned}
\|f\|^{2}+\left\|d_{A} f\right\|^{2} & =\|f\|^{2}+\left\|d_{A^{\prime}} f+\alpha \cdot f\right\|^{2} \\
& \leqq\|f\|^{2}+2\left(\left\|d_{A^{\prime}} f\right\|^{2}+\|\alpha\|_{\infty}^{2}\|f\|^{2}\right) \\
& \leqq\left(1+2\|\alpha\|_{\infty}^{2}\right)\left(\|f\|^{2}+\left\|d_{A^{\prime}} f\right\|^{2}\right)
\end{aligned}
$$

( $\|\cdot\|_{\infty}$ stands for the supremum-norm). Consequently, any two connections on $E$ define, locally (i.e. in some neighborhood of every given point in $M$ ), equivalent $L_{1}^{2}$-norms.

Finally, suppose $E \rightarrow M$ is $S$-equivariant, i.e., $S$ acts by bundle automorphisms preserving the connection and the metric on $E$ and $M$. Let $N$ be a tubular neighborhood of an orbit $S \cdot x$, and $i: D_{x} \hookrightarrow N$ the normal slice at $x$ (see Appendix A.2). Clearly, an $S$-invariant section of $E$ reduces, upon restriction, to an $S_{x}$-invariant section on $D_{x}$. This reduction is compatible with the $L_{1}^{2}$-norm in the following sense. 
Proposition A.1. Let $E \rightarrow M$ be an $S$-equivariant vector bundle. Then restricted to the set of $S$-invariant sections of $E$ over a tubular neighborhood $N$ of an $S$-orbit $S \cdot x \subset M$, the $L_{1}^{2}$-norm is equivalent, locally, to the $L_{1}^{2}$-norm over the normal slice at $x$; more precisely, there exists a tubular neighborhood $N^{\prime} \subset N$ and a constant $c>0$, such that for every $S$-invariant section $f$ of $E$ over $N^{\prime}$,

$$
\frac{1}{c}\left\|i^{*} f\right\|_{L_{1}^{2}\left(D_{x}^{\prime}\right)} \leqq\|f\|_{L_{1}^{2}\left(N^{\prime}\right)} \leqq c\left\|i^{*} f\right\|_{L_{1}^{2}\left(D_{x}^{\prime}\right)},
$$

where $i^{*} f$ is the restriction of $f$ to $D_{x}^{\prime}=D_{x} \cap N^{\prime}$.

Proof. Let $N^{\prime} \subset N$ be a tubular neighborhood of $S \cdot x$ with a compact closure in $N$ (such a neighborhood clearly exists). We first note that the statement in the proposition is true with $L_{1}^{2}$ replaced by $L^{2}$, as it follows immediately from the integration formula for an $S$-invariant function $f$ on $N$

$$
\int_{N} f=\int_{D_{x}} f \cdot \delta,
$$

where $\delta$ is a smooth positive function on $D_{x}$; namely, the determinant of the isomorphism (18) (up to a non-zero constant multiple).

For the $L_{1}^{2}$ case, the statement follows from the fact that, restricted to $S$ invariant sections, the difference between the covariant derivative $d_{A}$ on $N$ and the covariant derivative $d_{i^{*} A}$ along the slice is "tensorial" (a zero-order operator)

$$
d_{A}-d_{i^{*} A}=\phi \in v^{*}\left(D_{x}\right) \otimes \operatorname{End}(E),
$$

so the boundedness of $\phi$ on $N^{\prime}$ implies, as in (22), that the $L_{1}^{2}\left(N^{\prime}\right)$ norm, restricted to $S$-invariant sections, is equivalent to the $L_{1}^{2}\left(D_{x}^{\prime}\right)$ norm.

Acknowledgements. I wish to extend my deep gratitude to Jerry Maraden, my thesis supervisor, for his support, patience, and sound advice, and to Richard Montgomery, for suggesting the problem to me and helping all along. I would also like to thank Lorenzo Sadun for pointing out an error, now corrected, in the calculation concerning Remark 4 of Sect. 3.

\section{References}

1. Adams, J.F.: Lectures on Lie groups. Chicago: The University of Chicago Press 1969

2. Atiyah, M.F.: The geometry of Yang-Mills fields. Fermi Lectures, Scuola Normale Pisa, 1979

3. Atiyah, M.F., Bott, R.: A Lefschetz fixed point formula for elliptic complexes. I. Ann. Math. 86, 374-407 (1967)

4. Atiyah, M.F., Bott, R.: The moment map and equivariant cohomology. Topology 23 (No. 1), $1-28(1984)$

5. Atiyah, M.F., Hitchin, N.J., Singer, I.M.: Self-duality in four-dimensional Riemannian geometry. Proc. R. Soc. Lond. A 362, 425-461 (1978)

6. Bor, G., Montgomery, R.: SO (3)-invariant Yang-Mills fields which are not self-dual. In: Harnad, J., Marsden, J.E. (eds.) Hamiltonian systems, transformation groups, and spectral transform methods. Proceedings, Montreal, 1989. Montréal: Les publications CRM 1990

7. Bourguignon, J.-P., Lawson, H.B., Jr.: Stability and isolation phenomena for Yang-Mills fields. Commun. Math. Phys. 79, 189-230 (1981)

8. Kobayashi, S., Nomizu, K.: Foundations of differential geometry, Vol.I. New York: Interscience 1963

9. Parker, T.: Non-minimal Yang-Mills fields and dynamics. Preprint (1990)

10. Parker, T.: An equivariant Morse theory for Yang-Mills. Preprint (1991)

11. Palais, R.S.: The principle of symmetric criticality. Commun. Math. Phys. 69, 19-30 (1979) 
12. Sadun L., Segert, J.: Non-self-dual Yang-Mills connections with quadrupole symmetry. Commun. Math. Phys.

13. Sibner, L.M., Sibner, R.J., Uhlenbeck, K.K.: Solutions to Yang-Mills equations which are not self-dual. Proc. Nat1. Acad. Sci. USA 86, 8610 (1989)

14. Taubes, C.H.: On the Equivalence of the first and second order equations for gauge theories. Commun. Math. Phys. 75, 207-227 (1980)

15. Taubes, C.H.: Stability in Yang-Mills theories. Commun. Math. Phys. 91, 235-263 (1983)

16. Urakawa, H.: Equivariant theory of Yang-Mills connections over Riemannian manifolds of cohomogeneity one. Indiana Univ. Math. J 37, No. 4188, 753-788 (1988)

17. Uhlenbeck, K.K.: Connections with $L^{p}$ bounds on curvature. Commun. Math. Phys. 83, 3142 (1982)

Communicated by A. Jaffe 